\title{
Advances in epilepsy: new perspectives on new-onset epilepsy, comorbidities, and pharmacotherapy
} Andres M Kanner

\author{
Addresses: Rush Medical College at Rush University; Laboratory of Electroencephalography and Video-EEG-Telemetry; and Section of Epilepsy and \\ Rush Epilepsy Center, Rush University Medical Center, 1653 West Congress Parkway, Chicago, IL 60612, USA \\ Email: akanner@rush.edu
}

FI000 Medicine Reports 2010, 2:5I (doi:I0.34I0/M2-5I)

The electronic version of this article is the complete one and can be found at: http://fl000.com/reports/medicine/content/2/5 I

\begin{abstract}
The purpose of this brief article is to review old concepts of the significance of acute symptomatic seizures, the impact of psychiatric comorbidities on the response of pharmacologic and surgical treatments of the seizure disorder, and the importance of factoring comorbid medical comorbidities into the choice of antiepileptic drugs (AEDs). In addition, this article provides an update on the latest data on the teratogenic effects of AEDs and reviews the most relevant results of a recent practice guideline on pregnancy issues in women with epilepsy. The article closes with a review of the latest advances in the therapeutic effects of first- and second-generation AEDs.
\end{abstract}

\section{Introduction and context}

The approach to the evaluation and management of epilepsy has continued to evolve in the last two years. The purpose of this article is to highlight some of the more relevant advances with respect to the significance of a first seizure, the need to incorporate medical and psychiatric comorbidities, and advances in first- and second-generation antiepileptic drugs (AEDs).

\section{Recent advances \\ Reassessing old assumptions}

a) The good and bad news of a first acute symptomatic seizure In 2005, Fisher and colleagues [1] suggested a redefinition of epilepsy as having experienced at least one seizure associated with an enduring alteration of the brain that increases the likelihood of future seizures and neurobiologic, cognitive, and social disturbances. Four years later, Hesdorffer et al. [2] questioned the significance of a first acute symptomatic seizure in a study that compared the mortality and risk of seizure recurrence between 262 patients with acute symptomatic seizures caused by acute stroke, central nervous system (CNS) infection, or trauma and 148 patients with a first unprovoked seizure caused by static brain lesions. While patients with acute symptomatic seizures had an almost ninefold higher risk of dying within the first 30 days after the seizure, they were $80 \%$ less likely to have subsequent unprovoked seizures over the following 10 years compared with patients with a first unprovoked seizure.

b) Are cognitive deficits identified in patients with epilepsy the result of recurrent seizures?

The long-held belief that cognitive deterioration in patients with idiopathic/cryptogenic epilepsy is a consequence of recurrent seizures was revised in a recent population-based study of 155 newly diagnosed epilepsy patients in whom no CNS pathology could be identified. All patients underwent a neuropsychological evaluation before the start of AED therapy. Compared with 87 healthy volunteers from the general population who were matched for age and sex, patients with epilepsy (PWEs) performed significantly worse than healthy volunteers on 6 of 14 cognitive measures after adjustments for age, sex, and education. Of note, cognitive performance was not related to the number of seizures, type of epilepsy, or mood [3]. Thus, this study suggests that cognitive deficits in many patients 
with idiopathic/cryptogenic epilepsy are related to the CNS disturbances that resulted in the seizure disorder.

\section{Reassessing the significance of comorbidities: the treatment of epilepsy is not restricted to the remission of seizures}

In the last decade, epileptologists have recognized the importance of recognizing and managing comorbid medical and psychiatric disorders. Publications in the last two years reflect the increased interest in these problems.

\section{a) Psychiatric comorbidities}

Recent population-based studies have identified a high lifetime prevalence of depressive and anxiety disorders of up to $35 \%$ [4]. The negative impact of comorbid mood disorders on the quality of life in PWEs had already been recognized by several independent investigators in the last decade. In the last two years, the negative impact of comorbid mood disorders on the suicidal risk of PWEs has been recognized. In a population-based study, Christensen et al. [5] demonstrated that the risk to achieve completed suicide increases by 32 -fold in the presence of comorbid mood disorder compared with a twofold increased risk in the absence of any psychiatric comorbidity.

The negative impact of comorbid mood disorders on the response of pharmacologic and surgical treatment of seizures was reported in two recent studies. Hitiris et al. [6] found that a history of depression preceding the onset of epilepsy increased the risk of pharmaco-resistant epilepsy by twofold, and Kanner et al. [7] found that a lifetime history of depression was an independent predictor against achieving seizure freedom following an antero-temporal lobectomy.

Yet despite the relatively high prevalence of mood disorders in PWEs and the significant negative impact on their lives, no randomized controlled study had been conducted on its treatment in the last 30 years until recently, when Gilliam and colleagues [8] conducted a randomized controlled study comparing the efficacy of sertraline (up to $200 \mathrm{mg} /$ day) and cognitive behavior therapy in the treatment of major depressive episodes in 140 consecutive patients. The results presented at the 2009 annual meeting of the American Academy of Neurology (AAN) revealed symptom remission in $60 \%$ of patients randomly assigned to each treatment arm. Furthermore, patients assigned to sertraline did not have a worsening of seizures. Thus, these data demonstrate the efficacy of these two treatment modalities, which are comparable to those seen in people with primary major depressive episodes.
In January of 2008, the US Food and Drug Administration (FDA) issued an alert regarding the association between suicidality (defined as suicidal ideation, behavior, and completed suicides) and AEDs [9]. The alert was based on results of a meta-analysis that included data from 199 randomized clinical trials of 11 AEDs (carbamazepine, felbamate, gabapentin, lamotrigine, levetiracetam, oxcarbazepine, pregabalin, tiagabine, topiramate, valproate, and zonisamide). Suicidality occurred in 4.3 per 1000 patients treated with AEDs in the active arm and in 2.2 per 1000 patients in the comparison arm. Of all of the suicidality reported, suicidal ideation accounted for $67.6 \%$, preparatory acts for $2.8 \%$, attempts for $26.8 \%$, and completed suicide for $2.8 \%$. AEDs were found to be associated with a greater risk for suicidality with epilepsy (odds ratio [OR] 3.53, 95\% confidence interval [CI] 1.2812.10) than with psychiatric disorders (OR 1.51, 95\% CI 0.95-2.45) or other disorders (OR 1.87; 95\% CI 0.814.76). The FDA concluded that there was a statistically significant 1.80 -fold increased risk of suicidality with exposure to AEDs. Since its publication, this meta-analysis has been viewed as a source of great controversy and the validity of its results has been questioned by professional societies, including the American Epilepsy Society and the American Neurologic Association. In fact, a detailed review of the data of this meta-analysis demonstrated several serious methodological problems [10]. These included the following: (a) The assessment of suicidality was based on 'spontaneous' reports of patients and was not gathered in a systematic prospective manner. (b) The FDA associated the increased risk of suicide with all AEDs, despite the fact that statistical significance was found in only 2 (i.e., topiramate and lamotrigine) of the 11 AEDs studied. Furthermore, inclusion of three additional studies of lamotrigine resulted in the loss of statistical significance for this AED. Two other AEDs, valproic acid and carbamazepine, actually yielded a 'small protective effect'. The FDA's decision to present the risk as involving all AEDs stemmed from a concern that singling out specific AEDs might only change prescribing practices rather than emphasize the suicide risk. (c) Most epilepsy trials (92\%) included patients on adjunctive therapy (compared with $14 \%$ of psychiatric trials and $15 \%$ of other medical trials). It is unclear whether the higher suicidality rates in the epilepsy trials were due to drug interactions, given the high proportion of epilepsy trials designed with polytherapy, or whether they potentially were due to the low suicidality risk associated with carbamazepine and valproate - both drugs were protective for suicidality and were the most common comparison drugs in these trials. (d) Suicidal behavior was greater in certain geographic regions. For example, the ORs of suicidality were 1.38 (95\% CI 0.9-2.13) in North American studies and 4.53 (95\% CI 1.86-13.18) in studies conducted elsewhere. 
While there is no question that several AEDs can cause psychiatric adverse events, including suicidal ideation and behavior, the FDA's conclusions must be interpreted with great caution at this time. Only a study in which suicidality data are collected in a systematic and prospective manner can help determine whether a specific AED increases or decreases the risk. Yet because the FDA has decided to insert suicide warnings in the package inserts of all AEDs, these data cannot be disregarded; thus, physicians will need to identify patients with increased risks of suicide.

\section{b) Medical comorbidities}

Because they may have a negative impact, comorbid medical conditions must be considered in the choice of AEDs. For example, in 34 PWEs taking two of the most frequently prescribed enzyme-inducing AEDs (phenytoin and carbamazepine), hyperlipidemia and high C-reactive protein decreased significantly when switched to AEDs without enzyme-inducing properties (lamotrigine and levetiracetam) [11].

Osteoporosis and osteopenia have been recognized for a long time in patients treated with enzyme-inducing AEDs. A recent study, however, shows that significant decrease in bone mineral density can be identified after only 1 year of therapy. In a study of 93 premenopausal women who had been taking phenytoin monotherapy for 1 year, a significant decrease in bone mineral density in the head of the femur was found in women treated with phenytoin, but not in those on carbamazepine or lamotrigine [12]. Thus, while enzyme-inducing AEDs are the most widely prescribed drugs in the treatment of epilepsy, they should be used with great caution and avoided in patients at risk for cardiovascular disease and osteoporosis.

\section{c) Pregnancy in women with epilepsy}

Teratogenic impact of antiepileptic drugs

Teratogenic effects of valproic acid have been recognized for the last 10 years. However, this AED can negatively affect the cognitive development of children exposed to it in utero. In a recent study, Meador et al. [13] demonstrated that children exposed to valproic acid in utero had significantly lower IQ scores at 3 years of age than those who had been exposed to phenytoin, carbamazepine, and lamotrigine. Of note, in a practice guideline on pregnancy in women with epilepsy, the AAN recommended that AED serum concentrations be monitored (see below) [14].

Contradictory data on the risk of lamotrigine to cause cleft lip or cleft palate by two different pregnancy registries were published in 2008 . While neither registry found an overall risk of major malformations, the North American AED Pregnancy Registry found that an increased risk of orofacial clefts was 11.2-fold higher compared with their control group of 221,746 unexposed infants born at a Boston hospital [15]. On the other hand, the EUROCAT (European Registration of Congenital Anomalies) registry, which is a populationbased case control study that includes over one-quarter of births in Europe, did not find an increased risk of orofacial clefts for children exposed to lamotrigine monotherapy. Thus, this problem remains unsolved at this time [16].

In a practice guideline on the use of AEDs in pregnant women with epilepsy, the AAN made the following suggestions: (a) monotherapy should be used whenever possible, and any reductions of AED doses or conversion to monotherapy should be carried out before pregnancy. (b) Lowest doses should be used whenever possible, allowing for non-disabling simple partial seizures. (c) Avoid phenobarbital and valproic acid as much as possible [17].

By the same token, the AAN guidelines put into question the following long-held beliefs: (a) There is possibly a modestly increased risk of delivery by cesarean section and probably no substantially increased risk of late pregnancy bleeding. (b) There is probably no moderately increased risk of premature labor and delivery except for women who smoke [17].

Pharmacokinetic changes of antiepileptic drugs during pregnancy

The clearance of lamotrigine and levetiracetam, particularly that of lamotrigine, which doubles by the end of the third trimester, has been found to increase during the course of pregnancy $[18,19]$. Accordingly, monthly serum concentrations need to be obtained and doses adjusted to prevent a drop in their serum concentration from resulting in breakthrough seizures.

What's new about the efficacy of old antiepileptic drugs? The use of ethosuximide, valproic acid, and lamotrigine in childhood absence epilepsy had been taken for granted, but no head-to-head comparison had ever been conducted. Glauser et al. [20] recently reported the results of a National Institutes of Health-sponsored multicenter randomized double-blind trial in 453 children with childhood absence epilepsy. After 4 months of treatment, $53 \%$ and $58 \%$ of the children randomly assigned to ethosuximide and valproic acid, respectively, remained seizure-free compared with only $29 \%$ of children randomly assigned to lamotrigine [20]. However, children randomly assigned to valproic acid experienced a greater 
number of adverse events, suggesting that ethosuximide appears to be the drug of choice. This observation, however, is restricted to children who have not had generalized tonic-clonic seizures as ethosuximide does not have a therapeutic effect in these seizures.

\section{What's new about the efficacy of second-generation antiepileptic drugs?}

In a double-blind placebo-controlled study, levetiracetam monotherapy was found to be effective in juvenile myoclonic epilepsy [21]. In addition, in a double-blind multicenter randomized study, levetiracetam and controlled-release carbamazepine in monotherapy were found to be equally effective in the treatment of newonset partial or primary generalized tonic-clonic seizures; furthermore, more than $90 \%$ of responders remained seizure-free at low daily doses (400 mg/day of carbamazepine and $1000 \mathrm{mg} /$ day of levetiracetam) [22].

In a large population-based study (Standard and New Antiepileptic Drugs, or SANAD), the efficacies of lamotrigine, topiramate, oxcarbazepine, gabapentin, and carbamazepine were compared in the treatment of new-onset partial epilepsy; lamotrigine was considered to be significantly better than carbamazepine, gabapentin, and topiramate and had a non-significant advantage compared with oxcarbazepine. The authors concluded that 'lamotrigine is clinically better than carbamazepine, the standard drug treatment, for time to treatment failure outcomes and is therefore a cost-effective alternative for patients diagnosed with partial-onset seizures' [23].

In a separate study, valproic acid, topiramate, and lamotrigine were compared in the treatment of newonset idiopathic generalized epilepsy. Valproic acid and topiramate yielded comparable efficacies, which were superior to that of lamotrigine but valproic acid was better tolerated than topiramate. The authors concluded that 'valproic acid should remain the drug of first choice for many patients with generalized and unclassified epilepsies. However, because of known potential adverse effects of valproate during pregnancy, the benefits for seizure control in women of childbearing years should be considered' [24].

\section{Implications for clinical practice}

This article focuses on revised old concepts of epilepsy and established treatments as these are very relevant to the general neurologist and clinician who treats PWEs. A separate article is necessary to review the third generation of AEDs that have become available as well as non-pharmacologic therapies, including deep brain stimulation of thalamic nuclei and responsive neurostimulation systems, as well as the role of new diagnostic modalities used in the presurgical evaluation of patients with pharmaco-resistant epilepsy.

To summarize, in terms of reassessing old assumptions, there is now evidence to suggest that acute symptomatic seizures should not always be considered an expression of epilepsy and that cognitive deficits in many patients with idiopathic/cryptogenic epilepsy are related to the CNS disturbances that resulted in the seizure disorder.

When the significance of comorbidities is reassessed, psychiatric and medical conditions must always be considered in the choice of AEDs as they may have a negative impact. Enzyme-inducing AEDs should be avoided as much as possible in patients at risk for cardiovascular disease and osteoporosis, whereas lipid profiles should be obtained in patients taking these AEDs. Furthermore, when these AEDs are used, calcium and vitamin D supplements should be recommended to minimize the risk of osteopenia and osteoporosis, which should be monitored every year with DEXA (dual-energy X-ray absorptiometry) scans.

In pregnant women with epilepsy, new guidelines published by the AAN and the American Epilepsy Society have recommended that AED serum concentrations be monitored; in addition, monotherapy should be used whenever possible and any reductions of AED doses or conversion to monotherapy should be carried out before pregnancy. Furthermore, low doses should be used whenever possible, allowing for non-disabling simple partial seizures. Finally, phenobarbital and valproic acid should be avoided as much as possible.

Among the second-generation AEDs, studies have shown that lamotrigine is a cost-effective alternative for patients diagnosed with partial-onset seizures [21], whereas levetiracetam can be a good alternative for new-onset generalized tonic-clonic seizures and juvenile myoclonic epilepsy.

One recent study looking into old AEDs suggests that ethosuximide is the drug of choice for the treatment of childhood absence epilepsy; however, other options should be considered in children who also experience generalized tonic-clonic seizures as ethosuximide does prevent their occurrence. In addition, valproic acid remains the drug of first choice for men with idiopathic generalized epilepsies.

\section{Abbreviations}

AAN, American Academy of Neurology; AED, antiepileptic drug; CI, confidence interval; CNS, central nervous system; FDA, US Food and Drug Administration; OR, odds ratio; PWE, patient with epilepsy. 


\section{Competing interests}

The author has received research grants from Novartis, GlaxoSmithKline and Pfizer.

\section{References}

I. Fisher RS, van Emde Boas W, Blume W, Elger C, Genton P, Lee P, Engel J Jr: Epileptic seizures and epilepsy: definitions proposed by the International League Against Epilepsy (ILAE) and the International Bureau for Epilepsy (IBE). Epilepsia 2005, 46:470-2.

2. Hesdorffer DC, Benn EK, Cascino GD, Hauser WA: Is a first acute symptomatic seizure epilepsy? Mortality and risk for recurrent seizure. Epilepsia 2009, 50:1 102-8.

3. Taylor J, Kolamunnage-Dona R, Marson AG, Smith PE, Aldenkamp AP, Baker GA; SANAD study group: Patients with epilepsy: cognitively compromised before the start of antiepileptic drug treatment? Epilepsia 2009, 5 I:48-56.

4. Tellez-Zenteno JF, Patten SB, Jetté N, Williams J, Wiebe S: Psychiatric comorbidity in epilepsy: a population-based analysis. Epilepsia 2007, 48:2336-44.

5. Christensen J, Vestergaard M, Mortensen P, Sidenius P, Agerbo E: Epilepsy and risk of suicide: a population-based case-control study. Lancet Neurol 2007, 6:693-8.

6. Hitiris N, Mohanraj R, Norrie J, Sills GJ, Brodie MJ: Predictors of pharmacoresistant epilepsy. Epilepsy Res 2007, 75:192-6.

7. Kanner AM, Byrne R, Chicharro A, Wuu J, Frey M: A lifetime psychiatric history predicts a worse seizure outcome following temporal lobectomy. Neurology 2009, 72:793-9.

8. Gilliam FG, Black KJ, Carter J, Vahle V, Randall A, Sheline Y, Tsai W-Y, Lustman P: Depression and Health Outcomes in Epilepsy: A Randomized Trial. Presented at the $6 \mathrm{I}^{\text {st }}$ Annual Meeting of the American Academy of Neurology: 25 April - 02 May 2009; Seattle, Washington, USA.

9. US Department of Health and Human Services, Food and Drug Administration, Center for Drug Evaluation and Research, Office of Translational Sciences, Office of Biostatistics: Statistical Review and Evaluation: Antiepileptic Drugs and Suicidality. 2008.

[http://www.fda.gov/ohrms/dockets/ac/08/briefing/2008-4372b I-0IFDA.pdf]

10. Hesdorffer DC, Kanner AM: The FDA alert on suicidality and antiepileptic drugs: fire or false alarm? Epilepsia 2009, 50:978-86.

II. Mintzer S, Skidmore CT, Abidin CJ, Morales MC, Chervoneva I, Capuzzi DM, Sperling MR: Effects of antiepileptic drugs on lipids, homocysteine, and C-reactive protein. Ann Neurol 2009, 65:448-56.

FI000 Factor 6.0 Must Read

Evaluated by Andres Miguel Kanner 0I Jun 2009

12. Pack AM, Morrell MJ, Randall A, McMahon DJ, Shane E: Bone health in young women with epilepsy after one year of antiepileptic drug monotherapy. Neurology 2008, 70:1586-93.

13. Meador KJ, Baker GA, Browning N, Clayton-Smith J, CombsCantrell DT, Cohen M, Kalayjian LA, Kanner A, Liporace JD, Pennell PB, Privitera M, Loring DW; NEAD Study Group: Cognitive function at 3 years of age after fetal exposure to antiepileptic drugs. N Engl J Med 2009, 360:1597-605.

\section{Changes Clinical Practice}

FI000 Factor 6.4 Must Read

Evaluated by Michael Marsh 17 Apr 2009, Driss Moussaoui 29 Apr 2009

14. Harden CL, Meador KJ, Pennell PB, Hauser WA, Gronseth GS, French JA, Wiebe S, Thurman D, Koppel BS, Kaplan PW, Robinson JN, Hopp J, Ting TY, Gidal B, Hovinga CA, Wilner AN, Vazquez B, Holmes L, Krumholz A, Finnell R, Hirtz D, Le Guen C; American Academy of Neurology; American Epilepsy Society: Management issues for women with epilepsy-Focus on pregnancy (an evidence-based review): II. Teratogenesis and perinatal outcomes: report of the Quality Standards Subcommittee and Therapeutics and Technology Subcommittee of the American Academy of Neurology and the American Epilepsy Society. Epilepsia 2009, 50:1237-46.
15. Holmes LB, Baldwin EJ, Smith CR, Habecker E, Glassman L, Wong SL, Wyszynski DF: Increased frequency of isolated cleft palate in infants exposed to lamotrigine during pregnancy. Neurology 2008, 70:2152-8.

FI000 Factor 3.0 Recommended

Evaluated by Alison Pack 03 Oct 2008

16. Dolk H, Jentink J, Loane M, Morris J, de Jong-van den Berg LT; EUROCAT Antiepileptic Drug Working Group: Does lamotrigine use in pregnancy increase orofacial cleft risk relative to other malformations? Neurology 2008, 71:7|4-22.

FI000 Factor 3.0 Recommended

Evaluated by Alison Pack 10 Sep 2008

17. Harden CL, Hopp J, Ting TY, Pennell PB, French JA, Allen Hauser W, Wiebe S, Gronseth GS, Thurman D, Meador KJ, Koppel BS, Kaplan PW, Robinson JN, Gidal B, Hovinga CA, Wilner AN, Vazquez B, Holmes L, Krumholz A, Finnell R, Le Guen C; American Academy of Neurology; American Epilepsy Society: Management issues for women with epilepsy-Focus on pregnancy (an evidence-based review): I. Obstetrical complications and change in seizure frequency: Report of the Quality Standards Subcommittee and Therapeutics and Technology Assessment Subcommittee of the American Academy of Neurology and the American Epilepsy Society. Epilepsia 2009, 50:1229-36.

18. Pennell PB, Peng L, Newport DJ, Ritchie JC, Koganti A, Holley DK, Newman M, Stowe ZN: Lamotrigine in pregnancy: clearance, therapeutic drug monitoring, and seizure frequency. Neurology 2008, 70:2130-6.

FI000 Factor 6.0 Must Read

Evaluated by Andres Miguel Kanner 02 Jul 2008

19. Longo $B$, Forinash $A B$, Murphy JA: Levetiracetam use in pregnancy. Ann Pharmacother 2009, 43:1692-5.

20. Glauser TA, Cnaan A, Shinnar S, Hirtz DG, Dlugos D, Masur D, Clark PO, Capparelli EV, Adamson PC; Childhood Absence Epilepsy Study Group: Ethosuximide, valproic acid and lamotrigine in childhood absence epilepsy. N Engl J Med 20I0, 362:790-9.

Changes Clinical Practice

FI000 Factor 9.0 Exceptional

Evaluated by Gihan Tennekoon 18 Mar 2010

21. Noachtar S, Andermann E, Meyvisch P, Andermann F, Gough WB, Schiemann-Delgado J; NI66 Levetiracetam Study Group: Levetiracetam for the treatment of idiopathic generalized epilepsy with myoclonic seizures. Neurology 2008, 70:607-16.

FI000 Factor 6.0 Must Read

Evaluated by Roderick Duncan 18 Apr 2008

22. Brodie MJ, Perucca E, Ryvlin P, Ben-Menachem E, Meencke HJ; Levetiracetam Monotherapy Study Group: Comparison of levetiracetam and controlled-release carbamazepine in newly diagnosed epilepsy. Neurology 2007, 68:402-8.

23. Marson AG, Al-Kharusi AM, Alwaidh M, Appleton R, Baker GA, Chadwick DW, Cramp C, Cockerell OC, Cooper PN, Doughty J, Eaton B, Gamble C, Goulding PJ, Howell SJ, Hughes A, Jackson M, Jacoby A, Kellett M, Lawson GR, Leach JP, Nicolaides P, Roberts R, Shackley P, Shen J, Smith DF, Smith PE, Smith CT, Vanoli A, Williamson PR; SANAD Study group: The SANAD study of effectiveness of carbamazepine, gabapentin, lamotrigine, oxcarbazepine, or topiramate for treatment of partial epilepsy: an unblinded randomised controlled trial. Lancet 2007, 369: 1000-15.

24. Marson AG, Al-Kharusi AM, Alwaidh M, Appleton R, Baker GA, Chadwick DW, Cramp C, Cockerell OC, Cooper PN, Doughty ], Eaton B, Gamble C, Goulding PJ, Howell SJ, Hughes A, Jackson M, Jacoby A, Kellett M, Lawson GR, Leach JP, Nicolaides P, Roberts R, Shackley P, Shen J, Smith DF, Smith PE, Smith CT, Vanoli A, Williamson PR; SANAD Study group: The SANAD study of effectiveness of valproate, lamotrigine, or topiramate for generalised and unclassifiable epilepsy: an unblinded randomised controlled trial. Lancet 2007, 369:1016-26. 